\title{
COVID-19 times: Study protocol for rapid assessment of the situation of women and children in Bihar
}

UNICEF

Population Council Institute

Follow this and additional works at: https://knowledgecommons.popcouncil.org/departments_sbsr-pgy

Part of the Demography, Population, and Ecology Commons, International Public Health Commons, and the Public Health Education and Promotion Commons How does access to this work benefit you? Let us know!

\section{Recommended Citation}

UNICEF and Population Council Institute. 2020. "COVID-19 times: Study protocol for rapid assessment of the situation of women and children in Bihar," COVID-19 Study Methodology Brief. New Delhi: UNICEF and Population Council Institute. 


\section{STUDY PROTOCOL FOR RAPID ASSESSMENT OF THE SITUATION OF WOMEN AND CHILDREN IN BIHAR}

\section{BACKGROUND}

\begin{abstract}
India reported its first COVID-19 case on January 30, 2020 and numbers began to rise in late March 2020. The containment measures implemented by the national and state governments, particularly the nationwide lockdown, have helped keep the COVID-19 pandemic relatively at a low level in India.

- Bihar, being one of the poorest states in India, is particularly at high risk of adverse health and economic effects of COVID-19 and lockdown.

- The Population Council Institute, in partnership with UNICEF Bihar, is conducting a rapid longitudinal study that aims to inform the design and development of locally relevant suitable response mechanisms to increase resilience and thereby reduce vulnerabilities of local communities in Bihar.
\end{abstract}

\section{KEY RESEARCH QUESTIONS}

What are the circumstances and needs of women and children during the COVID19 crisis?

\section{Phase 1 focuses on the following:}

- Availability, access and utilisation of social protection and humanitarian actions in a variety of sectors such as health, nutrition, water, sanitation and hygiene (WASH), child protection and education, including online and extra school learning resources.

- Barriers and facilitators in availing social protection benefits.

- Characteristics of sub-population groups vulnerable to COVID-19 and/or health risks, with specific reference to maternal and child health, mental health, nutrition and WASH.

- Impact of the lockdown on livelihoods/income, basic needs, health, and measures taken, if any, by families, communities and systems to minimise these impacts.

- Effect of migration on women and children during the epidemic.

What is the immediate (soon after the post-lockdown) and long-term (five months post-lockdown) impact of the epidemic and related shocks such as loss of livelihoods on health and well-being, with particular focus on women and children and the effects of mitigation measures on key outcomes?

Phases 2 and 3 will help answer the following:

- Time trends in key outcomes, e.g. utilisation of maternal and child health services, WASH practices, mental health, schooling, and livelihood.

- Status of implementation of mitigation measures and effects among women and children

- The cycle of job loss and gain and migration during the epidemic (among sub-groups of study participants).

\section{RESEARCH DESIGN}

This is a three-phase longitudinal study to answer the above research questions. The plan is to gather perspectives of multiple stakeholders-young men and women and/or their adult family members, and influential adults in the community such as leaders of women's self-help groups (SHG), frontline workers (ASHAs, AWWs, and ANMs) and representatives of Panchayati Raj Institutions (PRI). Interviews with the potential participants will happen through phone or face to-face (whenever appropriate), using structured and semi-structured questionnaires or interview guides.

The plan is to conduct the study in three phases-

Phase 1 - during the lockdown (telephone survey)

Phase 2 - post-lockdown (telephone)

Phase 3 - five months post-lockdown (face-to-face/in-person survey)

Telephone surveys which are expected to take 15 minutes, will be conducted by a team of trained gender-matched research assistants.

The study uses Population Council-led three existing prospective cohort studies in Bihar; (1) a prospective cohort study of adolescents aged 10-19 years (Understanding the Lives of Adolescents and Young Adults (UDAYA); (2) a prospective cohort study of self-help groups; (3) a longitudinal study of frontline workers.

For interviews with representatives of PRIs and faith leaders, a conveniently drawn sample will be used, sourced from interviews with frontline workers and SHG leaders. The study was reviewed and approved by the Institutional Review Board of the Population Council.

\section{CONTACT INFORMATION}

This work was jointly undertaken by Population Council Institute and UNICEF/Bihar. For more information, contact nsaggurti@popcouncil.org;

\section{PHASE 1 DATA COLLECTION}

The phase 1 survey was conducted between May 13-25, 2020 through telephone interviews using 25 experienced research assistants (7-8 interviews/day per research assistant). Data was entered into a CSPro program loaded onto mini-laptops or smartphones. The questionnaire for the Phase 1 survey included select (and brief answer) questions on key thematic areas to answer the research questions. Interviews with young men or women took an average of 13 minutes. The participants interviewed and sample size achieved (including the non-response rates) are as follows:

\section{$\underline{\text { Household-level survey of young men and women and/or their household }}$}

members

- The total number of people approached for interviews were 1825 (1364 women and 461 men)-participants randomly selected from the UDAYA cohort.

A total of 794 participants (including 576 women and 218 men) consented and participated in the interview.

- The response rate was about $44 \%$ (42\% for women and $47 \%$ for men).

- The major reasons for non-response were: phone out of service/no incoming facility (66\%), did not pick up the call despite three calls or participant refused to participate in the survey (21\%), and other reasons (13\%).

\section{Survey of SHG leaders}

- A total of 299 SHG leaders were selected from three districts viz. Munger Patna and Rohtas. Of these, a total of 179 participants consented and participated in the interview (with a $60 \%$ response rate). The reasons for non-participation remained the same, with maximum telephone numbers being non-functional or no incoming facility (88\%).

\section{Survey of frontline workers (FLWs)}

- A total of 179 accredited social health activists (ASHAs) were selected from three districts viz. Munger, Patna, and Rohtas.

- Of these, 61 ASHAs consented and participated in the interview. The major reason for non-response was phone out of service or no incoming facility (87\%).

- During the Phase 1 survey with ASHAs, contact numbers of 44 Auxiliary Nurse Midwives (ANMs) and 34 Anganwadi Workers (AWWs) were obtained. Of these, 28 and 22 were interviewed, respectively.

\section{LIMITATIONS OF PHASE 1 SURVEY}

The results presented in thematic briefs may be interpreted with caution in the context of several limitations.

- Firstly, the response rate in the telephone survey ranged between $30 \%$ and $51 \%$, a comparison was done of those who were selected randomly from the UDAYA cohort for the COVID-19 study and those who could be interviewed in the COVID-19 study (see the table below). The comparison was made across some key characteristics. Results of such an analysis indicated that there is more representation of households with a higher standard of living in the COVID-19 study sample, given their ownership of phone and the number retention/portability. One can interpret this bias to the extent that all vulnerability indicators are the minimum estimates, and the situation may be much worse than those reported in this set of study briefs.

- Secondly, the survey was conducted during the first few days of Lockdown 4.0 in May 2020, around the time when migrants started returning home. Hence, not many migrant households received their family members back home before the end of the survey. Also, the government announced major social and monetary schemes around the same time, which may not have been fully captured in Phase 1 of the survey.

- Lastly, the limited sample size and selectivity of population groups limit the generalization. For example, the eligible sample size of participants for access to maternal health services was limited. Similarly, due to constraints of telephone surveys, not all relevant information could be captured in Phase 1 of the survey.

\begin{tabular}{|lccc|} 
Background characteristics & $\begin{array}{c}\text { Respondents not } \\
\text { included in Phase 1 } \\
\text { survey }\end{array}$ & $\begin{array}{c}\text { Respondents } \\
\text { interviewed in Phase 1 } \\
\text { survey }\end{array}$ & $\begin{array}{c}\text { Mean } \\
\text { Difference }\end{array}$ \\
\hline Years of education (mean) & 6.95 & 8.61 & $1.657^{* * *}$ \\
\hline $\begin{array}{l}\text { Completed upper primary (8+ years) } \\
\text { level of education }\end{array}$ & 55.5 & 79.3 & $23.8^{* * *}$ \\
\hline Currently in school (\%) & 63.6 & 68.3 & $4.7 * *$ \\
\hline Mothers level of education (mean) & 2.44 & 3.38 & $0.935 * * *$ \\
\hline Place of residence ( rural \%) & 52.7 & 51 & 1.7 \\
\hline Social group (\% SC\ST) & 23.2 & 17.4 & $5.8^{* * *}$ \\
\hline Religion (\% Hindu) & 83.6 & 85.6 & 2 \\
\hline Household wealth Score (mean) & 20.29 & 22.83 & $2.535^{* * *}$ \\
\hline Total number of respondents & 9639 & 794 & \\
\hline
\end{tabular}

